PROCEEDINGS OF THE

AMERICAN MATHEMATICAL SOCIETY

Volume 125, Number 6, June 1997, Pages 1685-1688

S 0002-9939(97)03643-5

\title{
A REMARK ON COMMUTING OPERATOR EXPONENTIALS
}

\author{
EDGAR M. E. WERMUTH
}

(Communicated by Theodore W. Gamelin)

\begin{abstract}
In a previous paper the author proved that for square matrices with algebraic entries $\exp (A) \exp (B)=\exp (B) \exp (A)$ if and only if $A B=B A$. This result is extended here to bounded operators on an arbitrary Banach space.
\end{abstract}

The simple and well-known fact that

$$
A B=B A \text { implies } e^{A} e^{B}=e^{B} e^{A}=e^{A+B},
$$

where $A$ and $B$ may be square matrices of equal order, bounded operators on a Banach space, or elements of a unical Banach algebra, has no obvious general converse.

An easy case is that of selfadjoint operators on a Hilbert space:

If $A$ and $B$ are bounded selfadjoint operators on a Hilbert space then

$$
e^{A} e^{B}=e^{B} e^{A} \text { if and only if } A B=B A
$$

moreover,

$$
e^{A} e^{B}=e^{A+B} \text { if and only if } A B=B A .
$$

To prove the 'only if' part of (1), observe that if $A$ is selfadjoint, then $e^{A}$ is positive, and the positive square root $e^{A / 2}$ can be approximated by a sequence of polynomials in $e^{A}$ (for a simple iterative construction of suitable polynomials, due to C. Visser, see, e.g., [8, p. 261f.], or [16, p. 222ff.]; a more general statement is $\left[4\right.$, Theorem 11.3.5]). Hence $e^{A} e^{B}=e^{B} e^{A}$ implies $e^{A / 2^{n}} e^{B / 2^{n}}=e^{B / 2^{n}} e^{A / 2^{n}}$ for all $n \in \mathbb{N}$. Expanding the power series on both sides leads to

$$
A B+O\left(2^{-n}\right)=B A+O\left(2^{-n}\right) \quad(n \rightarrow \infty),
$$

whence $A B=B A$.

The second equivalence is a trivial consequence of the first one, since $\left(e^{A} e^{B}\right)^{*}=$ $e^{B} e^{A}$.

Received by the editors May 9, 1995 and, in revised form, September 6, 1995.

1991 Mathematics Subject Classification. Primary 39B42, 47A60, 30E10.

Key words and phrases. Commuting exponentials, Dunford's integral, Runge's theorem.

For valuable comments I thank Heinrich Bock, Robert B. Burckel, Hans-Günter Meier, and the referee.

(c) 1997 American Mathematical Society 
There is a more general version of (1) in the matrix case: For square matrices of equal order and with algebraic entries ${ }^{1} e^{A} e^{B}=e^{B} e^{A}$ if and only if $A B=B A$ ([14, Theorem 1]; see also $[1,13,11]$ and the interesting surveys [2] and [12]; a thorough treatment of matrix exponentials is given in [5]). We now extend this result to arbitrary bounded operators on a Banach space.

Definition. We call a set $\Lambda$ of complex numbers $2 \pi i$-congruence-free, if there are no two different elements $\lambda_{1}, \lambda_{2} \in \Lambda$ such that $\lambda_{1} \equiv \lambda_{2}(\bmod 2 \pi i)$.

Theorem. Let $A$ and $B$ be bounded operators on a Banach space with $2 \pi i$-congruence-free spectra. Then

$$
e^{A} e^{B}=e^{B} e^{A} \text { if and only if } A B=B A .
$$

Remark. The restriction concerning the spectra of $A$ and $B$ cannot be dispensed with, as is seen by simple two-dimensional examples:

$$
A=\left(\begin{array}{cc}
0 & \pi \\
-\pi & 0
\end{array}\right) \quad \text { implies } e^{A}=-I,
$$

but there are normal matrices $B$ such that $A B \neq B A$, e.g.

$$
B=t\left(\begin{array}{ll}
1 & 1 \\
1 & 1
\end{array}\right)(t \in \mathbb{C} \backslash\{0\}) .
$$

Proof. Of course, we need only treat the 'only if' part.

Let $\sigma(A)$ denote the spectrum of $A$. Then

$$
(\sigma(A)+2 k \pi i) \cap \sigma(A)=\emptyset
$$

for $k \in \mathbb{Z} \backslash\{0\}$.

The spectrum $\sigma(A)$ being compact, there is an $\varepsilon>0$ such that with

$$
D:=U_{\varepsilon}(\sigma(A)):=\{z \in \mathbb{C}:|z-\lambda|<\varepsilon \text { for some } \lambda \in \sigma(A)\}
$$

we have

$$
D \cap \bigcup_{k \in \mathbb{Z} \backslash\{0\}}(D+2 k \pi i)=\emptyset .
$$

This implies that the mapping

$$
z \mapsto e^{z} \quad(z \in D)
$$

is injective whence it has a holomorphic inverse (e.g., [3, Corollary 5.78] or [9, Theorem 10.33])

$$
f: e^{D} \rightarrow D
$$

such that

$$
f\left(e^{z}\right)=z \quad(z \in D) .
$$

Now we choose a finite subcover $\left(B_{\varepsilon}(z):=U_{\varepsilon}(\{z\})\right)$

$$
\bigcup_{j=1}^{N} B_{\varepsilon / 2}\left(z_{j}\right)
$$

\footnotetext{
${ }^{1}$ In fact, the proof in [14] shows that the conclusion holds for arbitrary matrices with $2 \pi i$ congruence-free spectra (see below for a definition of this notion), algebraic entries being just an illustrative sufficient condition for this spectral property.
} 
of the open cover $\bigcup_{z \in \sigma(A)} B_{\varepsilon / 2}(z)$ of $\sigma(A)$; we put

$$
K:=\bigcup_{j=1}^{N} \overline{B_{\varepsilon / 2}\left(z_{j}\right)}
$$

This compact set $K$ has a boundary $\partial K$ consisting of finitely many closed Jordan curves with a positive distance from $\sigma(A)$, and

$$
\sigma(A) \subset K \subset D
$$

According to Dunford's functional calculus (see, e.g., [6, chap. 4], [15, p. 225ff.] and [10, chap. 10]) we thus have

$$
e^{A}=\frac{1}{2 \pi i} \int_{\partial K} e^{z}(z-A)^{-1} d z .
$$

From Runge's theorem (see, e.g., [3, chap. 8] or [9, chap. 13]) we infer the existence of a sequence $\left\{r_{n}\right\}$ of rational functions with poles outside $e^{K}$ such that $r_{n}(z) \rightarrow f(z)$ uniformly for $z \in e^{K}$. Writing

$$
r_{n}(z)=\frac{p_{n}(z)}{q_{n}(z)}
$$

where all zeros of the polynomials $q_{n}$ lie outside $e^{K}$, we conclude that $q_{n}\left(e^{A}\right)$ is invertible and $q_{n}\left(e^{z}\right)$ is holomorphic in a neighbourhood of $K$, and we have

$$
\begin{aligned}
& r_{n}\left(e^{A}\right)=p_{n}\left(e^{A}\right)\left(q_{n}\left(e^{A}\right)\right)^{-1} \\
& =\frac{1}{2 \pi i} \int_{\partial K} r_{n}\left(e^{z}\right)(z-A)^{-1} d z \\
& \left\|r_{n}\left(e^{A}\right)-A\right\| \rightarrow 0 \quad(n \rightarrow \infty) .
\end{aligned}
$$

An analogous reasoning concerning $B$ leads to a sequence $\left\{s_{n}\right\}$ of rational functions such that

$$
\left\|s_{n}\left(e^{B}\right)-B\right\| \rightarrow 0 \quad(n \rightarrow \infty)
$$

Since

$$
r_{n}\left(e^{A}\right) s_{n}\left(e^{B}\right)=s_{n}\left(e^{B}\right) r_{n}\left(e^{A}\right)
$$

the conclusion follows.

We implicitly assumed the Banach space to be a complex one; the extension of the theorem to real Banach spaces by means of complexification (see [7, chap. 1]) is trivial. Moreover, the theorem and its proof are valid for unical Banach algebras, too. But this is no true generalization, since any such algebra is isomorphic to an algebra of bounded operators on a Banach space (see [7, chap. 1] or [10, chap. 10]).

Even in the case of two-dimensional matrices the equivalence (2) does not hold under the weaker assumption of $2 \pi i$-congruence-free spectra ([14, p. 128]). Thus there remains an important open problem in the general case: To find a natural additional assumption (analogous to the one in the Theorem) which together with $e^{A} e^{B}=e^{A+B}$ implies $A B=B A$. 


\section{REFERENCES}

1. Dennis S. Bernstein, Problem 88-1, Commuting Matrix Exponentials, SIAM Rev. 30 (1988), 123.

2. L Some Open Problems in Matrix Theory Arising in Linear Systems and Control, Linear Algebra Appl. 162-164 (1992), 409-432. MR 94a:93017

3. Robert B. Burckel, An Introduction to Classical Complex Analysis, Vol. 1, Basel, 1979. MR 81d:30001

4. Einar Hille, Methods in Classical and Functional Analysis, Reading (Mass.), 1972. MR $\mathbf{5 7 : 3 8 0 2}$

5. Roger A. Horn and Charles R. Johnson, Topics in Matrix Analysis, Cambridge, 1991. MR 92e: 15003

6. Edgar R. Lorch, Spectral Theory, New York, 1962. MR 25:427

7. Charles E. Rickart, General Theory of Banach Algebras, Princeton, 1960. MR 22:5903

8. Frédéric Riesz et Béla Sz.-Nagy, Leçons d'Analyse Fonctionelle, Budapest, 1952.

9. Walter Rudin, Real and Complex Analysis, 3rd edition, New York, 1987. MR 88k:00002

10. _ Functional Analysis, 2nd edition, New York, 1991. MR 92k:46001

11. Wasin So, Equality Cases in Matrix Exponential Inequalities, SIAM J. Matrix Anal. Appl. 13 (1992), 1154-1158. MR 93j:15010

12. Robert C. Thompson, High, Low, and Quantitative Roads in Linear Algebra, Linear Algebra Appl. 162-164 (1992), 23-64. MR 92k:15006

13. Edgar M. E. Wermuth, Solution to Problem 88-1, SIAM Rev. 31 (1989), 125-126.

14. - Two Remarks on Matrix Exponentials, Linear Algebra Appl. 117 (1989), 127-132. MR 90e: 15019

15. Kôsaku Yosida, Functional Analysis, 6th edition, Berlin, 1980. MR 82i:46002

16. Nicholas Young, An Introduction to Hilbert Space, Cambridge, 1988. MR 90e:46001

Zentralinstitut für Angewandte Mathematik, Forschungszentrum Jülich GmbH, Postfach 1913, D-52425 Jülich, Germany

E-mail address: e.m.e.wermuth@kfa-juelich.de

Current address: FBAllgemeinwissenschaften und Informatik, Georg-Simon-Ohm-FH Nürnberg, Postfach 210320, D-90121 Nürnberg, Germany

E-mail address: edgar.wermuth@ai.fh-nuernberg.de 